\title{
Handling pandemic and seasonal diseases together: An unprecedented challenge to the Indian health care system

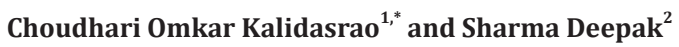 \\ ${ }^{1}$ Department of Clinical Biochemistry, Vardhman Mahavir Medical College (VMMC) \& Safdarjung Hospital, New Delhi-110029, India \\ ${ }^{2}$ Department of Community Medicine, Pramukhswami Medical College, Karamsad, Anand, Gujarat-388325, India
}

\begin{abstract}
*Corresponding author: Dr. Omkar Kalidasrao Choudhari, Department of Clinical Biochemistry, Vardhman Mahavir Medical College (VMMC) \& Safdarjung Hospital, New Delhi-110029, India. Tel.: +91-9958166730; Email: omkarchoudhari@yahoo.com
\end{abstract}

Received 3 December 2020; Revised 18 February 2021; Accepted 26 February 2021; Published 8 March 2021

Citation: Kalidasrao CO, Deepak S. Handling pandemic and seasonal diseases together: An unprecedented challenge to the Indian health care system. J Med Sci Res. 2021; 9(2):54-55. DOI: http://dx.doi.org/10.17727/JMSR.2021/9-e1

Copyright: (C) 2021 Kalidasrao CO et al. Published by KIMS Foundation and Research Center. This is an open-access article distributed under the terms of the Creative Commons Attribution License, which permits unrestricted use, distribution, and reproduction in any medium, provided the original author and source are credited.
Around a year back corona virus disease-19 (COVID19), then recognised as SARS CoV 2 was started in the Wuhan province of China [1]. It spreaded all over the world and was declared pandemic by WHO in March 2020 [2]. India stood at $2^{\text {nd }}$ place worldwide in number of affected individuals just below United States of America [3]. The prolonged period of 9 months of worldwide COVID-19 in absence of effective vaccine and mortality reaching a million globally, the healthcare system is burdened with a huge quantum of patients. While India is fighting the pandemic in the most efficient way, the seasonal diseases like dengue fever, malaria, chikungunya fever are also on rise [4].

Cases of dengue fever has maintained its constant presence in national capital, Delhi since long. Every year in the months of July to October, the admissions pertaining to dengue fever, malaria or chikungunya fever see a rising trend. In 2010, there was outbreak of dengue fever in Delhi and the disease caused substantial morbidity, hospital admissions and mortality [5].

In the presence of few similar signs and symptoms like fever, malaise in dengue fever, malaria, chikungunya fever and COVID-19, [6] avoidance to access healthcare system in the light of COVID-19, many cases might be underreported and may not receive appropriate treatment. The social stigma raised during this pandemic has led to hesitancy among citizens to approach healthcare system. The 
possibility of airborne spread of corona virus have been demonstrated in some literature which adds on to the anxiety of the patients with the possibility of getting infected without coming in contact with the case and reluctance to visit the hospital [7]. This anxiety causes not only delay in diagnosis, but also affect the mental health of the individual, moreover patients suffering from other co morbidities have an intense fear of getting diseased and with no regular follow up for their existing ailments, chances of getting sick per se because of their comorbidities are increased. Government is taking serious steps towards handling of this issue via introduction of telemedicine guidelines. However, this service should reach to public which is in despair due to COVID-19 pandemic because of loss of jobs, loss of savings in the lockdown period and also beam of hope for any vaccine is meagre as many trials are paused [8].

Dengue fever is caused by family of virus Flaviviridae, generally presented with fever, malaise, body ache, bleeding from any site. The complication of dengue haemorrhagic fever (DHF) occur in $3 \%$ of cases of dengue fever while the mortality in DHF is up to $5 \%$ with shock and no specific treatment is available apart from symptomatic management, hence early detection is imperative [9]. Dengue fever and COVID19 , both the diseases exhibit coagulation abnormality. The thrombocytopenia has poor prognosis in both dengue haemorrhagic fever (DAH) and in COVID-19. Mortality in dengue is mainly associated with third space oedema, hypotension, bleeding diathesis, circulatory shock while in COVID-19 available literature suggest role of micro thromboembolism in patients [10]. While mortality in both the diseases are mainly seen in initial days, irrespective of best management mortality remains high (In DHF and COVID-19).

Reports of COVID-19 and dengue fever coexistence has been also reported and could have severe outcome due to conglomeration of both the virus presenting with both the bleeding and clotting diathesis, presenting a big challenge to the physicians [10, 11]. Moreover, government medical establishments are already burdened with the number of patients and all people do not have medical insurance, hence affordability for treatment in private institutions is an another big issue.

So, the present scenario is bringing a constant stress on the Indian healthcare system. Measures like establishing separate fever clinics, contact tracing, private isolation facilities, mandatory quarantine for persons with history of contact with community surveillance are few measures government is taking for the effective containment of the pandemic. However, with increase in number of the diseased persons with COVID-19 every day, despite strict steps by the government and in the absence of an effective vaccine, following proper preventive measures remain the most vital step.

\section{Conflict of interest}

There are no conflicts of interest.

\section{References}

[1] Huang C, Wang Y, Li X, Ren L, Zhao J, et al. Clinical features of patients infected with 2019 novel coronavirus in Wuhan, China. Lancet. 2020; 395(10223): 497-506.

[2] Cucinotta D, Vanelli M. WHO Declares COVID-19 a Pandemic. Acta Biomed. 2020; 91(1):157-160.

[3] WHO Coronavirus Disease (COVID-19) Dashboard. Covid19. who.int. 2020 [cited 15 November 2020]. Available from: https://covid19.who.int/.

[4] Delhi: 99 dengue cases in a week take tally to 831 | Delhi News - Times of India. The Times of India. 2020 [cited 28 November 2020]. Available from: https://timesofindia. indiatimes.com/city/delhi/delhi-99-dengue-cases-in-aweek-take-tally-to-831/articleshow/79276721.cms

[5] Ahmed NH, Broor S. Dengue Fever outbreak in Delhi, north India: a clinico-epidemiological study. Indian J Community Med. 2015; 40(2):135-138.

[6] Chikungunya, dengue or Covid? Ahmedabad Mirror. September 19 2020, [cited 28 November 2020]. Available from: https://ahmedabadmirrorindiatimes.com/ ahmedabad/others/chikungunya-dengue-or-covid/ articleshow/78197288.cms

[7] Morawska L, Milton DK. It is time to address airborne transmission of Coronavirus Disease 2019 (COVID-19). Clin Infect Dis. 2020; 71(9): 2311-2313.

[8] NPR Choice page. Nprorg. 2020; [cited 30 October 2020]. Available from: https://www.npr.org/sections/coronaviruslive-updates/2020/09/08/910863680/covid-19-vaccinetrial-paused-due-to-illness-in-one-volunteer.

[9] Monath TP. Dengue: the risk to developed and developing countries. Proc Natl Acad Sci USA.1994; 91:2395-2400.

[10] Connors J, Levy J. COVID-19 and its implications for thrombosis andanticoagulation. Blood.2020; 135(23):20332040.

[11] Verduyn M, Allou N, Gazaille V, Andre M, Desroche T, et al. Co-infection of dengue and COVID-19: A case report. PLoS Negl Trop Dis. 2020; 14(8):e0008476. 